\title{
SOME PROPERTIES OF WEIGHTED HYPERBOLIC POLYNOMIALS
}

\author{
TETSUO INOUE \\ Department of Applied Mathematics \\ Kobe Mercantile Marine College \\ Higashinada, Fukae-Minami \\ Kobe, JAPAN
}

(Received May 21, 1992 and in revised form January 16, 1995)

\begin{abstract}
A new notion of weighted hyperbolic polynomials is introduced and their properties are discussed in this paper.

KEY WORDS AND PHRASES. Weighted hyperbolic polynomial and capacity, weak convergence, normalized counting measure on zeros.
\end{abstract}

1991 AMS SUBJECT CLASSIFICATION CODE. 30E10, 41A10.

\section{INTRODUCTION.}

Theorems in approximation theory have been studied by a lot of researchers. In $[1,2,3,4,5,6,7$, $8,12,13$ ] the cases for the weighted polynomials (shorty $w$-polynomials) in the complex plane were discussed, and in $[9,10]$ the cases for ones in locally compact spaces are also considered.

In the note we will introduce the definition of weighted hyperbolic polynomials (shortly $w \cdot h$ polynomials) in the unit disk and discuss their properties.

Almost all of the theorems with respect to $w \cdot h$-polynomials in the unit disk are proved by a simple modification or the methods analogous to the cases for weighted ones in the complex plane or locally compact spaces. Therefore we omit the proof but a few exceptions.

The introduction of the definition of $w h$-polynomials gives the following advantages:

(1) Theorems (propositions) on $w \cdot h$-polynomials can be easily obtained in the form similar to ones on usual algebraic polynomials. An example asserts that $w \bullet h$-capacity, transfinite diameter and Chebyshev constant are equal to each other.

(2) The introduction makes it easy to verify or apply theorems. For example, the sharpness of inequalities can be easily verified. The property will be shown for the case of the finite-infinite-range inequality.

The outline of the note is as follows. In Section 2 , definitions of $w \bullet h$-capacity, transfinite diameter, Chebyshev constant and polynomials are introduced. Furthermore we discuss the definitions of normalized counting measure of zeros of $w \bullet h$-polynomials and the weak convergence.

In Section 3 we show that some propositions on $w \cdot h$-polynomials have the same form as the usual ones. The sharpness of some inequality is also discussed

In Section 4 a theorem on the normalized counting measure on zeros of $w \cdot h$-polynomials is presented. We then give a part of proof as the application of proposition in Section 3, which shows that the introduction of the definition of $w \cdot h$-polynomials are useful for the application.

2. DEFINITIONS

Let $E$ be a compact set in the unit disk $U=\{|z|<1\}$ and $D$ be a domain whose boundary consists of $\gamma_{0}=\{|z|=1\}$ and $\partial E$, where $\partial E$ is the outer boundary of $E$. 
The positive, bounded weight function $w(z)$ and $E$ satisfy each of the following assumptions:

(1) $\log w$ is a continuous function on $U$.

(2) $E$ has positive $w \cdot h$-capacity.

At first we state the definitions of $w \bullet h$-capacity and transfinite diameter which is a translation [6]. Let $M(E)$ denote the class of all positive unit Borel measures whose support is contained in $E$. Also, for any function $f: E \rightarrow C$, we set

$$
\|f\|_{E}=\sup _{z \in E}|f(z)|
$$

We want to define the modified capacity of $E$. So we define the weighted hyperbolic energy of $\sigma \in M(E)$ by

$$
I_{w}(\sigma)=-\iint \log \left[\left|\frac{z-t}{1-\bar{t} z}\right| w(z) w(t)\right] d \sigma(z) d \sigma(t)
$$

Using the notation

$$
V_{w}=V(w, E)=\sup _{\sigma \in M(E)}\left\{-I_{w}(\sigma)\right\}
$$

the $w \cdot h$-capacity of $E$ is given by

$$
C p h(w, E)=\exp \left(V_{w}\right) .
$$

Let $S_{w}=\operatorname{support}\left(\mu_{w}\right)$, where $\mu_{w} \in M(E)$ is an extremal measure such that $I_{w}\left(\mu_{w}\right)=V_{w}$. The existence of $\mu_{w}$ can be shown analogously [see 6, Theorem 3.1(b)].

For an integer $n \geq 2$, we set

$$
\begin{aligned}
& \delta_{n}(w, E)=\sup _{z_{1}, \ldots, z_{n} \in E}\left\{\prod_{1 \leq i<j \leq n}\left[\left|\frac{z_{i}-z_{j}}{1-\bar{z}_{j} z_{i}}\right| w\left(z_{i}\right) w\left(z_{j}\right)\right]\right\}^{2 /(n(n-1))} \\
& =\left\{\prod_{1 \leq i<j \leq n}\left[\left|\frac{z_{i}^{*}-z_{j}^{*}}{1-\bar{z}_{j}^{*} z_{i}^{*}}\right| w\left(z_{i}^{*}\right) w\left(z_{j}^{*}\right)\right]\right\}^{2 /(n(n-1))}
\end{aligned}
$$

where $\left\{z_{i}^{*}\right\}_{i=1}^{n}$ is called the $w \cdot h$-Fekete points.

Then we define the $w \cdot h$-transfinite diameter of $E$ by

$$
\operatorname{Trh}(w, E)=\lim _{n \rightarrow \infty} \delta_{n}(w, E),
$$

where the convergence of the sequence $\left\{\delta_{n}(w, E)\right\}$ can be shown analogously as (see [6]).

Next, we introduce a new notion of $w \cdot h$-polynomials. For each integer $n \geq 1$, we let $P_{n, w}$ denote the class of all polynomials

$$
p_{n, w}(z)=\prod_{i=1}^{n}\left[\left(\frac{z-z_{i}}{1-\bar{z}_{i} z}\right) w(z) w\left(z_{i}\right)\right]
$$

for $z, z_{i} \in U$, which we call the $w \bullet h$-polynomials of degree $n$. To modify the $w$-Chebyshev constant introduced in [6], we consider

$$
\alpha_{n}(w, E)=\inf _{p_{n, w} \in P_{n, w}}\left\|p_{n, w}(z)\right\|_{E}
$$

A standard argument shows that the infimum in (2.8) is attained for some $p_{n, w}^{*}(z)$, which is called a $w \cdot h$-Ch-polynomial. Note that, for integers $n, m \geq 1$,

$$
\alpha_{n+m}(w, E) \leq \alpha_{n}(w, E) \alpha_{m}(w, E),
$$


and then the sequence $\left\{\alpha_{n}(w, E)^{1 / n}\right\}$ converges.

Corresponding to the definition of the $w$-Chebyshev constant introduced in [6], the (new) modified Chebyshev constant is defined by

$$
C h h(w, E)=\lim _{n \rightarrow \infty} \alpha_{n}(w, E)^{1 / n}
$$

We remark that Mhaskar and Saff have studied the weighted polynomials

$$
\prod_{i=1}^{n}\left[\left(z-z_{\imath}\right) w(z)\right]
$$

instead of

$$
\prod_{\imath=1}^{n}\left[\left(\frac{z-z_{\imath}}{1-\bar{z}_{\imath} z}\right) w(z) w\left(z_{\imath}\right)\right]
$$

The notion of the $w \bullet h$-polynomials is motivated from (2.5), that is, "the $w \bullet h$-distance between the points $z_{i}$ and $z_{j}$ " must be

$$
\left|\frac{z_{\imath}-z_{\jmath}}{1-\bar{z}_{\jmath} z_{\imath}}\right| w\left(z_{\imath}\right) w\left(z_{\jmath}\right)
$$

and moreover $w \cdot h$-capacity, transfinite diameter and Chebyshev constant must be equal to each other.

For $w \cdot h$-polynomials $p_{n, w}(z)$ of degree $n$, the discrete unit measure defined on compact sets in $U$ with mass $(1 / n)$ at each zero of $p_{n, w}(z)$ will be denoted by $\mu_{n, w}=\mu\left(p_{n, w}\right)$. It will be called the normalized counting measure on the zeros of $p_{n, w}(z)$. If $p_{n, w}(z)$ has multiple zeros, the obvious modification will be made.

The weak convergence of $\nu_{n}$ to $\nu$ as $n \rightarrow \infty$ is defined by

$$
\lim _{n \rightarrow \infty} \int f d \nu_{n}=\int f d \nu
$$

for every continuous function in the complex plane $C$ with compact support [11].

Note that $E$ is of positive capacity at each point if $\operatorname{cap}(\{\zeta \in E ;|z-\zeta|<\delta\})>0$ for every $z \in E$ and every $\delta>0$.

\section{PROPOSITIONS}

As an example, the proposition for the weighted hyperbolic case has the same form as the classical one, we will show the following with respect to $w \cdot h$-capacity, transfinite diameter and Chebyshev constant. It will be verified by a simple modification of results in $[6,9]$. Therefore we omit the proof.

PROPOSITION 3.1. There hold the equalities

$$
C p h(w, E)=\operatorname{Trh}(w, E)=C h h(w, E) .
$$

The next proposition is of great importance for the study of weighted polynomial approximation theory, which is essentially the same as [6,9]. However, we prefer the form different from [6,9], because it is simple and makes it easy to verify the sharpness of some inequality. Furthermore, the form is useful for the application, which will be shown in the next section.

PROPOSITION 3.2. For an arbitrary $w \bullet h$-polynomial

$$
p_{n, w}(z)=\prod_{\imath=1}^{n}\left[\left(\frac{z-z_{\imath}}{1-\bar{z}_{\imath} z}\right) w(z) w\left(z_{\imath}\right)\right]
$$




$$
g_{w}(z)=\exp \left\{\int \log \left[\left|\frac{z-t}{1-\bar{t} z}\right| w(z) w(t)\right] d \mu_{w}(t)\right\},
$$

the following inequality holds:

$$
\left|p_{n, w}(z)\right| \leq \frac{\left\|p_{n, w}(z)\right\|_{S_{w}}^{*}}{C p h(w, E)^{n}} g_{w}(z)^{n}
$$

for any $z$ in $D$, where $\left\|p_{n, w}(z)\right\|_{S_{w}}^{*}$ is the smallest number that is an upper bound for $\left|p_{n, w}(z)\right|$ q.e. on $S_{w}$. q.e. on $S_{w}$ means that it holds on $S_{w}$ if the subset $S_{w}^{\prime}$ of $S_{w}$ where it does not hold is capacity zero.

Since the proof is analogous to that in [6,9], so we omit it.

The sharpness of the inequality is easily shown from the well-known fact as follows: For the $w$ - $h$-polynomials

$$
p_{n, w}^{*}(z)=\prod_{i=1}^{n}\left[\left(\frac{z-z_{i}^{*}}{1-\bar{z}_{i}^{*} z}\right) w(z) w\left(z_{i}^{*}\right)\right]
$$

with the $w \cdot h$-Fekete points $\left\{z_{\imath}^{*}\right\}_{\imath=1}^{n},\left\{p_{n, w}^{* 1 / n}(z)\right\}$, and $\left\{\left\|p_{n, w}^{*}(z)\right\|_{S_{w}}^{* 1 / n}\right\}$ converges locally uniformly to $g_{w}(z)$ in $D$, and $C p h(w, E)$ respectively.

Proposition 3.2 can be restated in the following, which is called a finite-infinite-range inequality.

PROPOSITION 3.3. For an arbitrary $w \bullet h$-polynomial $p_{n, w}(z)$, the following inequality holds:

$$
\left\|p_{n, w}(z)\right\|_{K}^{1 / n} \leq \frac{\left\|g_{w}(z)\right\|_{K}}{C p h(w, E)}\left\|p_{n, w}(z)\right\|_{S_{w}}^{* 1 / n}
$$

for any compact set $K \subset U \backslash S_{w}$.

We note that Mhaskar [8] has obtained the following:

PROPOSITION 3.4. For an arbitrary w-polynomial $q_{n, w}(z)$, the following inequality holds:

$$
\left\|q_{n, w}(z)\right\|_{K} \leq c_{1} \exp \left(c_{2} n\right)\left\|q_{n, w}(z)\right\|_{S_{v}}^{*}
$$

for any compact set $K \subset C \backslash S_{w}$, where $c_{1}$ and $c_{2}$ are constants depending only on $w, E, K$. where

Considering the above Proposition 3.3, it can be easily shown that $c_{1}=1$ and $c_{2}=\log \frac{\left\|h_{v}(z)\right\|_{K}}{\operatorname{Cap}(w, E)}$,

$$
h_{w}(z)=\exp \left\{\int \log [|z-t| w(z) w(t)] d \nu_{w}(t)\right\}
$$

and $\nu_{w}$ is the extremal measure for $w$-capacity.

\section{APPLICATIONS}

Proposition 3.2 in the previous section plays an important role in many theorems. In this section we discuss an example that the proposition can be applied for the proof of some theorems.

We consider the case where

$$
\limsup _{n \rightarrow \infty}\left|p_{n, w}(z)\right|^{1 / n} \leq g_{w}(z)
$$

holds for q.e. $z \in S_{w}$.

Then we have

$$
\limsup _{n \rightarrow \infty}\left\|p_{n, w}(z)\right\|_{S_{w}}^{* 1 / n} \leq C p h(w, E) .
$$

This follows from the equality

$$
g_{w}(z)=C p h(w, E)
$$

for q.e. $z \in S_{w}$, which was shown in $[6,9]$ for the case of weighted capacity. Since the proof is similar, we omit it.

Using Proposition 3.2 in Section 3 and inequality (4.2), we have 


$$
\limsup _{n \rightarrow \infty}\left|p_{n, w}(z)\right|^{1 / n} \leq g_{u^{\prime}}(z)
$$

for all $z \in D$.

Letting $z$ tend to 1 in (43), we have

$$
\limsup _{n \rightarrow \infty} \frac{1}{n} \sum_{i=1}^{n} \log w\left(z_{\imath}\right) \leq \int \log w(t) d \mu_{u^{\prime}}(t) .
$$

Summing up, we have proved the following

PROPOSITION 4.1. If the inequality

$$
\limsup _{n \rightarrow \infty}\left|p_{n, w}(z)\right|^{1 / n} \leq g_{w}(z)
$$

holds for q.e. $z \in S_{w}$, then we have

$$
\limsup _{n \rightarrow \infty} \frac{1}{n} \sum_{\imath=1}^{n} \log w\left(z_{\imath}\right) \leq \int \log w(t) d \mu_{w}(t) .
$$

Using the above result, as an application of Proposition 3.2 we can prove the following:

THEOREM 4.2. Under the assumption that
(a) the zeros of $p_{n, w}(z)$ are restricted on $S_{w}$.
(b) $\log w(z)$ is contimuous on $E$,
(c) E is of positive capacity at each point,
(d) if the following inequalities hold:

$$
\limsup _{n \rightarrow \infty}\left|p_{n, w}(z)\right|^{1 / 2} \leq g_{w}(z)
$$

for q.e. $z \in S_{w}$ and

$$
\liminf _{n \rightarrow \infty} \frac{1}{n} \sum_{\imath=1}^{n} \log w\left(z_{\imath}\right) \geq \int \log w(t) d \mu_{w}(t)
$$

then $\mu_{n, w}$ converges weakly to $\mu_{w}$ as $n \rightarrow \infty$.

Combining (4.6) and (4.8), we also obtain

$$
\lim _{n \rightarrow \infty} \frac{1}{n} \sum_{i=1}^{n} \log w\left(z_{i}\right)=\int \log w(t) d \mu_{w}(t) .
$$

Since we intend to show how Proposition 3.2 is applied for the proof, the other part of the proof is omitted. The details will appear in a future paper.

The theorem also shows an example that the theorem on usual algebraic polynomials is translated to one on $w \cdot h$-polynomials.

We next give a well-known theorem of $w \cdot h$-polynomials which satisfies the condition of Theorem 4.2 as follows.

THEOREM 4.3. Let

$$
p_{n, w}^{*}(z)=\prod_{\imath=1}^{n}\left[\left(\frac{z-z_{\imath}^{*}}{1-\bar{z}_{\imath}^{*} z}\right) w(z) w\left(z_{\imath}^{*}\right)\right]
$$


be Chebyshev w•h-polynomials of degree $n$ whose zeros are restricted on $S_{w}$, that is,

$$
\left\|p_{n, w}^{*}(z)\right\|_{S_{w}}=\inf _{p_{n, w} \in P_{n, w}^{\prime}}\left\|p_{n, w}(z)\right\|_{S_{w}},
$$

where $P_{n, w}^{\prime}$ is the class of $w \bullet h$-polynomials whose zeros are restricted on $S_{w}$.

Then $\mu_{n, w}^{*}$ converges weakly to $\mu_{w}$ as $n \rightarrow \infty$, where $\mu_{n, w}^{*}$ is the normalized counting measure on the zeros of $p_{n, w}^{*}(z)$

\section{REFERENCES}

[1] BLATT, E.P., SAFF, E.B. and SIMKANI, M., Jentzsch-Szegö type theorems for the best approximations, J. London Math. Soc., 38 (1988), 307-316.

[2] GONCHAR, A.A. and RAKHMANOV, E.A., Equilibrium measure and the distribution of zeros of extremal polynomials, Mat. Sb. 125 (1984), 119-130.

[3] GÓRSKI, J., Sur certaines Propriétés de points extrémaux liés à un domaine plan, Ann. Polnici Math. 3 (1956), 32-36.

[4] KLEINER, W., Sur les approximations de M. Leja dans le problème plan de Dirichlet, Ann. Polonici Math. 15 (1964), 203-209.

[5] MHASKAR, H.N. and SAFF, E.B., Where does the sup norm of a weighted polynomial live? Constr. Approx. 1 (1985), 71-91.

[6] MHASKAR, H.N. and SAFF, E.B., Weighted analogous of capacity, transfinite diameter and Chebyshev constant, Const. Approx. 8 (1992), 105-124.

[7] MHASKAR, H.N. and SAFF, E.B., The distribution of zeros of asymptotically extremal polynomials, J. Approx. Theor. 65 (1991), 279-300.

[8] MHASKAR, H.N., Finite-infinite-range inequalities in the complex plane, Intern. J. Math. \& Math. Sci., 14 (1991), 625-368.

[9] MHASKAR, H.N., Weighted polynomials, radial basic functions and potentials on locally compact spaces, Numer. Func. Anal. and Optim. 11 (1990-1991), 987-1017.

[10] OHTSUKA, M., On potential in locally compact spaces, J. Sci. Hirosima Univ., ser. A-1 (1961), 135-352.

[11] LANDKOF, N.S., Foundation of Modern Potential Theory, Springer Verlag, 1972.

[12] SICIAK, J., Some applications of the method of extremal points, Colloq. Math. 11 (1964), 209250.

[13] STAHL, H. and TOTIK, V., General Orthogonal Polynomials, Cambridge University Press, 1990. 


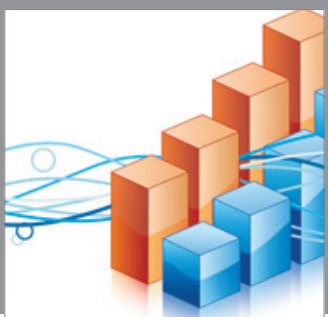

Advances in

Operations Research

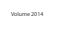

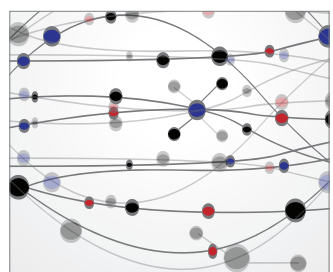

\section{The Scientific} World Journal
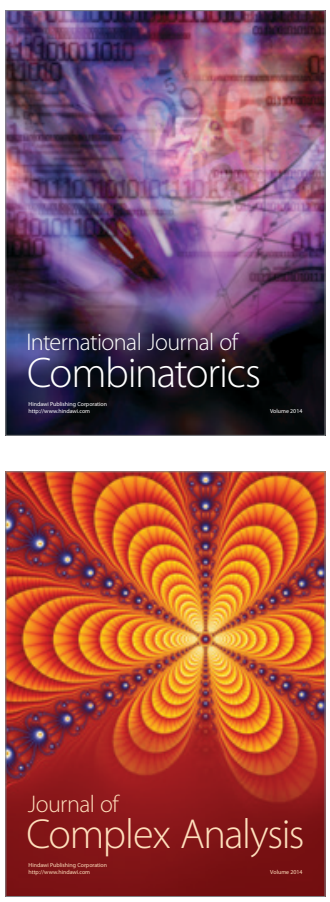

International Journal of

Mathematics and

Mathematical

Sciences
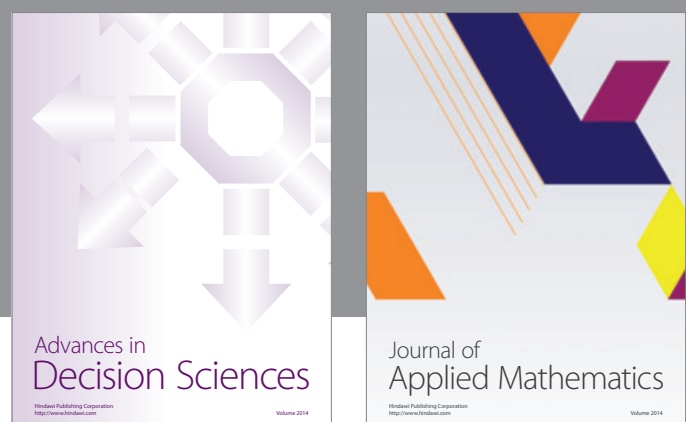

Journal of

Applied Mathematics
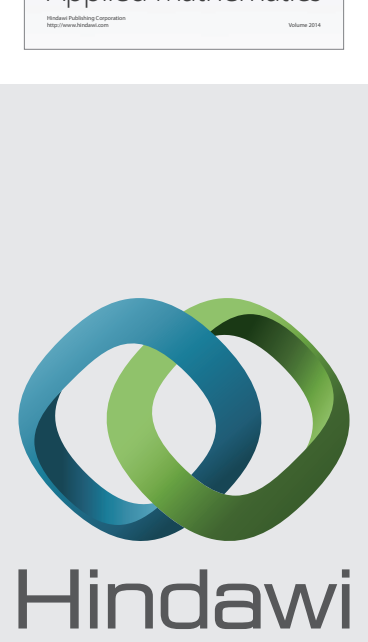

Submit your manuscripts at http://www.hindawi.com
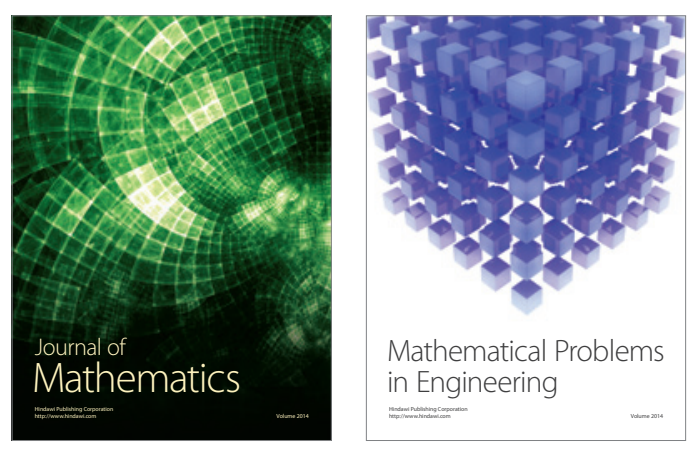

Mathematical Problems in Engineering
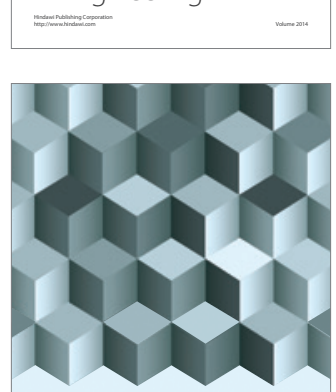

Journal of

Function Spaces
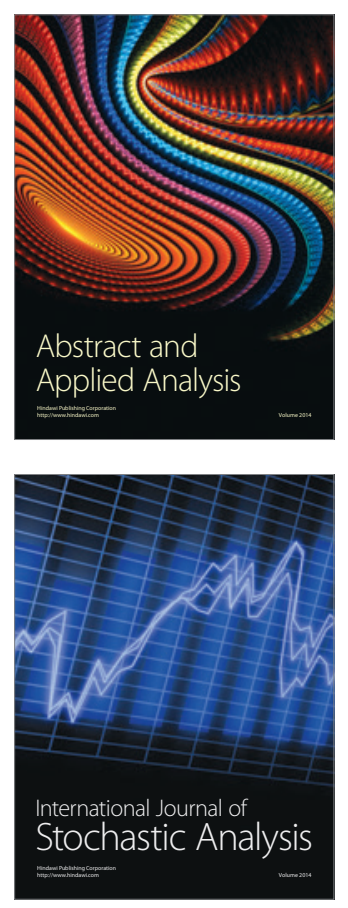

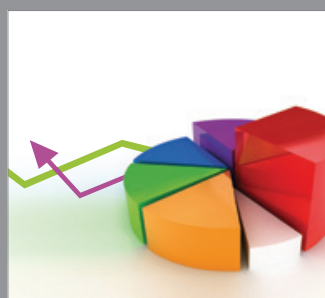

ournal of

Probability and Statistics

Promensencen
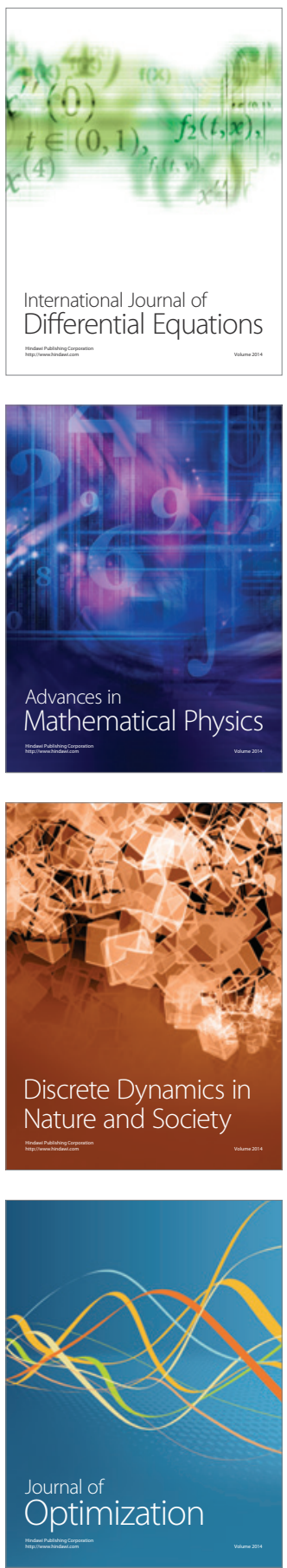